\title{
A Multidisciplinary Team Approach to the Treatment of Liver Cirrhosis
}

\author{
Yue-Rong Zhang' \\ Hui Wang ${ }^{2}$ \\ Ning Zhou ${ }^{2}$ \\ Yao-Di Zhang ${ }^{1,2}$ \\ Yan Lin ${ }^{1,2}$ \\ Li-Yang $\mathrm{Wu}^{1,2}$ \\ Shi-Fang Wei ${ }^{2}$ \\ Yan-Yun $\mathrm{Ma}^{2,3}$ \\ Chun-Xia Wang ${ }^{3}$ \\ 'Department of Gastroenterology, \\ University-Town Hospital of Chongqing \\ Medical University, Chongqing, People's \\ Republic of China; ${ }^{2}$ Department of \\ Infectious Diseases, The First People's \\ Hospital of Lanzhou, Gansu, 730050, \\ People's Republic of China; ${ }^{3}$ Department \\ of Laboratory, The First People's Hospital \\ of Lanzhou, Gansu, 730050, People's \\ Republic of China
}

Objective: To explore the feasibility of treating cirrhosis using a multidisciplinary team approach (MDT) and to pinpoint the key factors influencing its implementation.

Methods: The data of 307 patients with decompensated cirrhosis were studied retrospectively. Patients who received more than two treatment measures were assigned to the MDT group ( $\mathrm{n}=228$ ), and patients who received symptomatic medical drug treatment only were assigned to the traditional treatment group $(\mathrm{n}=79)$. The follow-up period ranged from 4 to 10 years, and the average follow-up period was 5.7 years. The results of the biochemical tests for hepatitis B virus deoxyribonucleic acid, hepatitis $\mathrm{C}$ virus ribonucleic acid, and autoantibodies to liver disease were analyzed.

Results: The differences in gender and Child-Pugh grade of liver function between the two groups were not statistically significant. The MDT group had obvious advantages over the traditional treatment group in occupational composition, etiology composition, 5-year survival rate and annual hospitalization times. The leading causes of death in the MDT group, in descending order, were liver cancer, infection, mesenteric thrombosis, and non-hepatic disease, and, in the medical treatment group, they were liver failure, gastrointestinal bleeding, infection, and liver cancer. There was a significant statistical difference between the two groups $(\mathrm{p}<0.05)$. In the multidisciplinary treatment, etiological treatment was the most widely used treatment, accounting for $79.8 \%$, followed by endoscopic treatment $(33.3 \%)$, peritoneal drainage and ascites reinfusion (25\%), splenectomy combined with devascularization $(11.4 \%)$ and stem cell transplantation and liver transplantation $(1.8 \%)$.

Conclusion: An MDT can improve the efficacy and prognosis of patients with cirrhosis and improve patient compliance. After multi-disciplinary intervention, the mortality spectrum of long-term survival patients with cirrhosis changes, and the mortality rate of liver cancer and non-liver disease increases

Keywords: liver cirrhosis, decompensated period, multidisciplinary team mode, traditional treatment, retrospective study

\section{Introduction}

Liver cirrhosis is a chronic progressive liver disease, and it is a common clinical condition resulting from liver damage. ${ }^{1}$ Most of the cirrhosis in patients in China is caused by hepatitis, but there is also a certain amount of alcoholic cirrhosis. ${ }^{2}$ In histopathology, cirrhosis is characterized by the extensive necrosis of hepatocytes, nodular regeneration of the remaining hepatocytes, structural destruction of hepatic lobules, and the formation of pseudo lobules, which finally develops into cirrhosis. ${ }^{3}$ In the early stage of cirrhosis, there may be no obvious symptoms due to the strong compensatory function of the liver, while in the later stages, the main
Correspondence: Yue-Rong Zhang Department of Gastroenterology, University-Town Hospital of Chongqing Medical University, No. 55 University Town Middle Road, Shapingba District, Chongqing, 40133I, People's Republic of China

$\mathrm{Tel} / \mathrm{Fax}+86$ I023657I4I42

Email zhang_yue_rong@I63.com 
manifestations are liver function damage, portal hypertension, and the involvement of multiple systems. In the advanced stage, upper gastrointestinal hemorrhaging, hepatic encephalopathy, secondary infection, ascites, and canceration may occur. ${ }^{3}$ At present, there is no way to cure cirrhosis. The purpose of any treatment is mainly to detect and prevent the progress of the disease in the early stages and carry out symptomatic treatment of its complications, including nutritional support, liver protection treatment, antiviral treatment, and portal vein pressure reduction treatment. $^{4,5}$ Although there are many potential forms of treatment for cirrhosis complications, especially for portal hypertension, they all have their advantages and disadvantages, ${ }^{4,5}$ and how best to deliver individualized treatment with hierarchical management, according to the clinical characteristics of the patient, requires further practical research.

A multi-disciplinary team approach (MDT) to treatment refers to when a group of multi-disciplinary experts discuss a patient and develop the best treatment plan for the patient based on their specialist opinions, making it a patient-centered, individualized approach. ${ }^{6}$ The MDT has been widely used in Europe and America and is playing an increasingly important role in the treatment of cancer and other diseases. ${ }^{6}$ Although the MDT has also been introduced and studied in major hospitals in China, it is not yet a perfect system, and in the treatment of decompensated cirrhosis, there are no MDT guidelines. Since individual differences in patients with cirrhosis are obvious, it is clear that a unified treatment model is not suitable for all patients.

Therefore, taking into account the current relevant diagnosis and treatment guidelines for cirrhosis, as well as clinical practice, this study explores the feasibility of the MDT and the key factors that may affect its implementation. Its objective is to improve the quality of life and prolong the survival of patients with liver cirrhosis, and to build a systematic, accurate, and scientific management model for chronic liver diseases, such as liver cirrhosis.

\section{Materials and Methods Subjects}

The data of 307 patients with decompensated cirrhosis were retrospectively analyzed. There were 192 males and 115 females, and their age range was 24-81 years old, with an average age of 56.2. The patients were followed up for 4-10 years, with an average follow-up of 5.7 years. Of these 307 patients, 146 had hepatitis B-induced cirrhosis, 61 had hepatitis C-induced cirrhosis, 23 had autoimmune cirrhosis, 22 had primary biliary cirrhosis, 28 had alcoholic cirrhosis, and 27 had cryptogenic cirrhosis. The diagnoses were based on the Guidelines for the Prevention and Treatment of Chronic Hepatitis B, Guidelines for the Prevention and Treatment of Hepatitis C (updated in 2015), Consensus on the Diagnosis and Treatment of Primary Biliary Cirrhosis (2015), Consensus on the Diagnosis and Treatment of Autoimmune Hepatitis (2015), and Guidelines for the Diagnosis and Treatment of Alcoholic Liver Disease (2015), ${ }^{4-6}$ which were jointly formulated by the Hepatology and Infectious Diseases branches of the Chinese Medical Association in 2010. The exclusion criteria included patients with primary liver cancer, acute liver failure, and human immunodeficiency virus infection.

The Ethics Committee of the First People's Hospital of Lanzhou approved the study, and all the patients provided signed informed consent. The livers that we transplanted for patients included were procured from organ donation after citizen death. All livers were donated voluntarily with written informed consent, and that this was conducted in accordance with the Declaration of Istanbul.

\section{Research Methods}

All the patients were tested at baseline for hepatitis $\mathrm{B}$ virus deoxyribonucleic acid (HBV DNA), hepatitis $\mathrm{C}$ virus ribonucleic acid (HCV RNA) (domestic reagents and imported reagents), six items of auto-liver disease immune antibodies, immunoglobulin, hepatitis series, four items of coagulation and alpha-fetal protein, and they also underwent virus genotyping, abdominal computed tomography, portal vein imaging, and gastroscopy. At 4, 12, and 24 weeks of the antiviral treatment, the biochemical and HBV DNA/ HCV RNA loads were retested. Once the HBV DNA load rebounded during the treatment, the resistance of nucleoside analogues was measured. The biochemical test was performed using an automatic biochemical detector, and the load of HBV DNA/HCV RNA was determined using Cobas AmpliPrep/COBAS TaqMan v 2.0, (Roche, Basel, Switzerland). The lower limit of detection value was HBV DNA $<20 \mathrm{IU} / \mathrm{mL}$ and HCV RNA $<15 \mathrm{IU} / \mathrm{mL}$. Real-time quantitative polymerase chain reaction technology was used for virus genotyping and nucleoside drug resistance testing, and the testing was assisted by the Gansu Jinyu Inspection Center. 


\section{The Multidisciplinary Diagnosis, Treatment, and Follow-Up of Liver Cirrhosis}

The multi-disciplinary diagnosis and treatment team consists of 15 experts from digestive liver disease, intervention, general oncology, endocrinology, pharmacy and other related majors The diagnosis and treatment procedure took place as follows: the patient was admitted to the Department of Digestive Liver Disease, and the team was responsible for the patient's etiology, endoscopic treatment, ascites drainage, and ascites reinfusion, and follow-up. The application was made by the Department of Digestive Liver Diseases, according to the needs of the patient, and the medical department was responsible for organizing the relevant experts to conduct multidisciplinary discussions to develop an individualized, combined, or sequential treatment strategy for the patient. If the patient needed a liver transplantation, they were transferred to a more advanced hospital, but the postoperative followup was still carried out by the hospital department. At the same time, follow-up files were established for the patient, who was reminded to come to the hospital for regular follow-ups by means of email, SMS, or telephone. Figure 1 shows the multidisciplinary diagnosis and treatment approach that was implemented. Different measures were taken for each patient in line with their specific symptoms and etiologies, be they portal hypertension, portal vein thrombosis, primary liver cancer, or liver dysfunction, for example. Figure 2 shows the follow-up procedure. The basic principle was to carry out relevant examinations every one to three months to see if there was any change in the disease, and the diagnosis and treatment process was adjusted as necessary.

\section{Grouping Standard}

During the treatment, the patients who received more than two forms of treatment were assigned to the MDT group $(\mathrm{n}=228)$, while the patients who received symptomatic medical drug treatment only, ${ }^{7}$ that is, medical liverprotecting, symptomatic, and diuretic drugs, and any

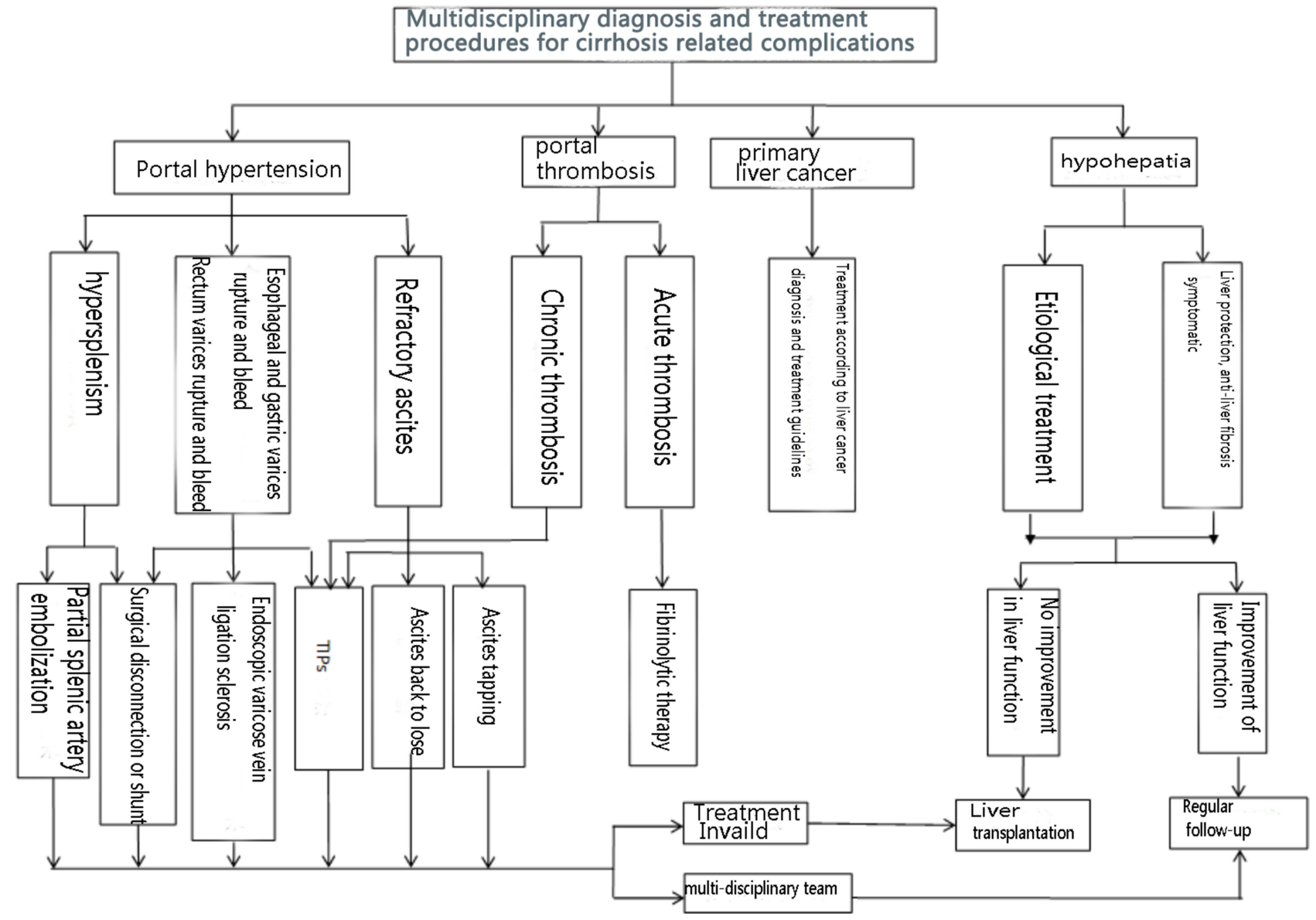

Figure I A roadmap for multidisciplinary comprehensive treatment. 


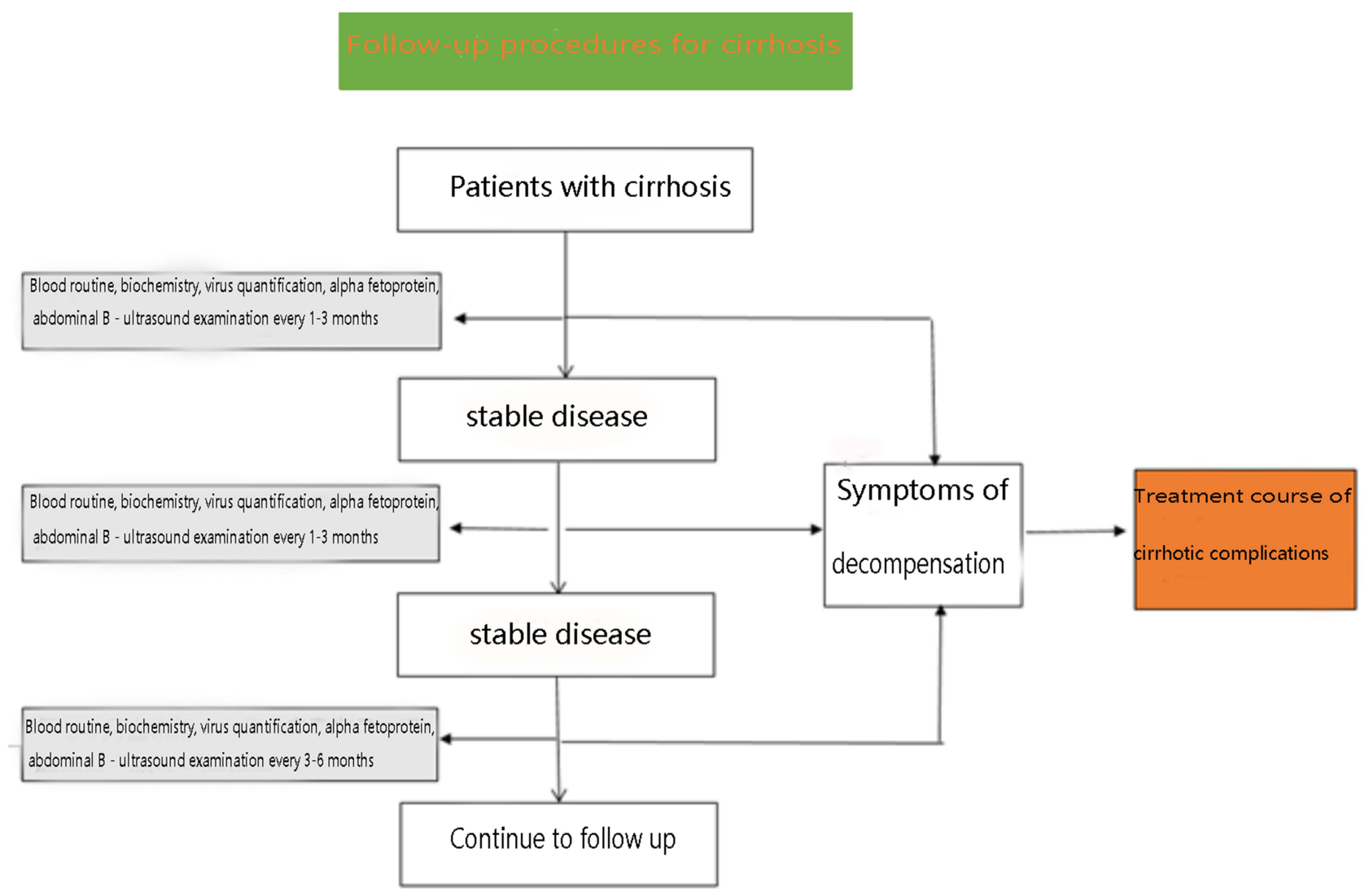

Figure 2 A roadmap for the follow-up management of cirrhosis.

patients who did not receive regular antiviral drugs, were assigned to the traditional treatment group $(n=79)$.

The clinical treatment included the following: ${ }^{8}$ (1) endoscopic treatment (consisting of esophageal varices ligation, sclerotherapy, and gastric fundus tissue glue injection,[endoscopic varix ligation/endoscopic injection sclerosis]); (2) transjugular intrahepatic portosystemic shunt (TIPS); (3) surgical splenectomy and devascularization; (4) partial splenic artery embolization (PSE); (5) ascites drainage; (6) stem cell transplantation; (7) liver transplantation; and (8) etiological treatment, which involved regular antiviral therapy, the specific monotherapy schemes being lamivudine, entecavir, or tenofovir fumarate or combined treatment with lamivudine and adefovir dipivoxil. The anti-HCV treatment regimens were sofosbuvir and velpatasvir or sofosbuvir and daclatasvir.

\section{Statistical Analysis}

The data were analyzed using statistical software SPSS19.0. Measurement data were expressed as mean \pm standard deviation $(x \pm \mathrm{SD})$. The intergroup comparison of the mean was conducted using a $t$-test. The proportions between the two groups were compared using a $X^{2}$ test. A p-value $<0.05$ was considered statistically significant.

\section{Results}

\section{The Comparison of Baseline Data Between the Two Groups}

Table 1 shows the baseline data of the MDT group and the traditional treatment group. The differences between the two groups in gender and Child-Pugh grade of liver function were not statistically significant.

However, the MDT group had obvious advantages over the traditional treatment group in terms of occupational composition, etiology composition, 5-year survival rates, and annual hospitalization times. In terms of occupational composition, workers accounted for nearly half of the patients in the MDT group, and farmers accounted for most of the patients in the traditional treatment group. In terms of etiological composition, the number of patients with hepatitis B-induced cirrhosis and hepatitis $\mathrm{C}$-induced cirrhosis ranked first and second in both groups, while alcoholic cirrhosis and cryptogenic cirrhosis ranked third in the MDT group, and autoimmune hepatitis cirrhosis 
Table I The Baseline Data of the MDT Group and the Traditional Treatment Group

\begin{tabular}{|c|c|c|c|c|}
\hline Variable & MDT Group & Traditional Treatment Group & Test value $\left(t / x^{2}\right)$ & $P$ \\
\hline Number of patients & 228 & 79 & & \\
\hline Male/Female & $142 / 86$ & $50 / 29$ & 0.677 & 0.496 \\
\hline Age (years) & $54 \pm 13.9$ & $56 \pm 11.5$ & 2.382 & 0.347 \\
\hline \multicolumn{5}{|l|}{ Occupational composition } \\
\hline Cadre & $39(17.1)$ & I & 27.539 & 0.000 \\
\hline Worker & $104(45.6)$ & 27 & & \\
\hline Farmer & $85(37.3)$ & 51 & & \\
\hline \multicolumn{5}{|l|}{ Etiology composition } \\
\hline Hepatitis B virus-related cirrhosis & II3 (49.5) & $33(4 I .8)$ & 9.215 & 0.101 \\
\hline Hepatitis $C$ virus-related cirrhosis & $49(21.5)$ & $12(15.2)$ & & \\
\hline Alcoholic cirrhosis & $20(8.8)$ & $8(10.1)$ & & \\
\hline Autoimmune hepatitis cirrhosis & $12(5.3)$ & II (I3.9) & & \\
\hline Primary biliary cirrhosis & $14(6.1)$ & $8(10.1)$ & & \\
\hline Cryptogenic cirrhosis & $20(8.8)$ & $7(8.9)$ & & \\
\hline Child-Pugh score of liver function & $8.1 \pm 2.6$ & $7.9 \pm 3.2$ & 0.782 & 0.335 \\
\hline 5-year survival rate $(\%)$ & $167 / 228(73.2)$ & $35 / 79(44.3)$ & 21.838 & 0.000 \\
\hline Annual hospitalization times & $1.8 \pm 0.3$ & $2.9 \pm 1.2$ & 2.345 & 0.026 \\
\hline \multicolumn{5}{|l|}{ Follow-up compliance } \\
\hline Well & $115 / 228(50.5)$ & 9/79 (11.4) & 87.274 & 0.000 \\
\hline Common & $74 / 228(32.4)$ & I2/79 (I5.2) & & \\
\hline Poor & $39 / 228(17.1)$ & $58 / 79(73.4)$ & & \\
\hline
\end{tabular}

Abbreviation: MDT, multi-disciplinary team.

ranked third in the traditional treatment group. In the MDT group, the 5-year survival rate was significantly higher than it was in the traditional treatment group $(\mathrm{p}<0.001)$, and the number of hospitalizations per year was significantly lower than it was in the traditional treatment group $(\mathrm{p}<0.05)$. The follow-up compliance of patients was significantly superior in the MDT group than it was in the traditional treatment group $(\mathrm{p}<0.001)$.

\section{The Selection and Implementation of the Multidisciplinary Comprehensive Treatment Measures}

Table 2 shows that, in terms of multidisciplinary treatment, etiological treatment was the most widely used treatment measure, accounting for $79.8 \%$ of patients, followed by endoscopic treatment $(33.3 \%)$, peritoneal drainage and ascites reinfusion $(25 \%)$, and splenectomy combined with devascularization (11.4\%), while stem cell transplantation and liver transplantation had the lowest proportion with $1.8 \%$. In addition, $24.6 \%$ of the patients received two treatments, and $75.4 \%$ received three or more treatments.

\section{Five-Year Mortality and Cause of Death in the Two Groups}

Table 3 shows that the 5 -year mortality rate in the MDT group was lower than it was in the traditional treatment group (26.8\% and $55.7 \%$, respectively). The causes of death in the MDT group were liver cancer, infection, mesenteric thrombosis, and non-liver disease, while the causes of death in the traditional treatment group were liver failure, gastrointestinal bleeding, infection, and liver cancer. There was a significant statistical difference in the ratio of death causes between the two groups $(p<0.05)$.

\section{Discussion}

Cirrhosis complications are a group of clinical syndromes that may manifest with multiple organ and system involvement, and, at present, there is no way to cure cirrhosis. Since the number of cirrhosis patients in China is large, the need for liver transplantations cannot be met. This means there is an urgent need to solve practical clinical problems through multidisciplinary comprehensive treatment strategies, for early detection and the prevention of the 
Table 2 Selection and Implementation of Multidisciplinary Comprehensive Treatment Measures and Patient Acceptance

\begin{tabular}{|c|c|c|}
\hline Multidisciplinary Treatment & Associated Complications & $\begin{array}{l}\text { Number of Patients } \\
\text { Treated(Ratio\%) }\end{array}$ \\
\hline $\begin{array}{l}\text { Endoscopic therapy(EVL/EIS/ } \\
\text { PTVE) }\end{array}$ & Esophagogastric variceal hemorrhage & $76(33.3)$ \\
\hline PSE & Hypersplenism/or associated bleeding & $10(5.3)$ \\
\hline TIPS & $\begin{array}{l}\text { Esophageal and gastric varices rupture hemorrhage + splenomegaly, } \\
\text { hypersplenism/refractory ascites + splenomegaly, hemorrhage }\end{array}$ & $5(2.2)$ \\
\hline $\begin{array}{l}\text { Splenectomy + decompression/ } \\
\text { shunt surgery }\end{array}$ & $\begin{array}{l}\text { Bleeding from esophageal and gastric varices + splenomegaly and hypersplenism } \\
\text { (no control after endoscopic treatment) }\end{array}$ & $26(11.4)$ \\
\hline Stem cell transplantation & Liver function synthesis ability is poor, liver function Child-Pugh score $\geq 9$ points & $4(1.8)$ \\
\hline Liver transplantation & Hepatic failure & $4(1.8)$ \\
\hline Peritoneal drainage & Refractory Ascites & $57(25)$ \\
\hline Etiological treatment & Antiviral, immunosuppressive agents, UDCA, abstinence treatment & 182(79.8) \\
\hline $\begin{array}{l}\text { General medicine of internal } \\
\text { medicine }\end{array}$ & Anti - infection, symptomatic, support & $228(100)$ \\
\hline \multicolumn{3}{|c|}{ The number/ratio of combined/sequential treatments for two or more treatments } \\
\hline Received two therapies & & $56 / 228(24.6)$ \\
\hline Received three therapies & & $125 / 228(54.8)$ \\
\hline Received four therapies & & $47 / 228(20.6)$ \\
\hline
\end{tabular}

Table 3 Causes of Death of Cirrhosis Patients in the Two Groups in 5 Years

\begin{tabular}{|l|l|l|}
\hline & MDT Group(n=228) & Traditional Treatment Group(n=79) \\
\hline Number of Patient Deaths at 5 Years (n) and Mortality Rate(\%) & $61(26.8)$ & $44(55.7)$ \\
\hline Cause of Death & & \\
Liver Cancer & $13(21.3)$ & $7(15.9)$ \\
Alimentary Tract Hemorrhage & $5(8.2)$ & $10(22.7)$ \\
Infection & $11(18.0)$ & $8(18.2)$ \\
Hepatic Failure & $8(13.1)$ & $13(29.55)$ \\
Portal Vein or Mesenteric Thrombosis & $9(14.8)$ & $2(4.55)$ \\
Hepatorenal Syndrome & $6(9.8)$ & $2(4.55)$ \\
Non-Hepatic Factors & $9(14.8)$ & $2(4.55)$ \\
\hline$X^{2}$ & 81.480 & \\
$P$ & 0.000 & \\
\hline
\end{tabular}

Abbreviation: MDT, multi-disciplinary team.

progression of the disease, to improve the prognosis and the quality of life of cirrhosis patients. Over the past ten years, relevant guidelines and the consensus concerning treatment have been constantly updated and refined with the support of evidence-based medicine, both home and abroad. The first and second levels for the prevention and treatment schemes of esophageal and gastric variceal hemorrhaging have been established. ${ }^{9}$ For patients with acute bleeding, failure after standard treatment for bleeding, complicated portal vein thrombosis, refractory ascites, or an absence of response to the hepatic venous pressure gradient, TIPS is recommended. ${ }^{10}$ When a patient has 
hypersplenism, the traditional methods have included surgical resection and PSE. ${ }^{10}$ Because this complication is one of the manifestations of portal hypertension, and it is not a clinical emergency, in recent years, there has been little research into it, and there have been no guidelines for its treatment. It is recommended, therefore, that if patients have bleeding and refractory ascites as a result of hypersplenism, their treatment should be either PSE combined with endoscopic treatment, TIPS, or surgery. This study has revealed that when using a multidisciplinary approach, etiological treatment was the most widely used form of treatment, followed by endoscopic treatment, peritoneal drainage and ascites reinfusion, splenectomy combined with devascularization, and stem cell transplantation, as well as liver transplantation. It is clear that the characteristics of the disease, the patient's individual requirements, local medical technology levels, the economy, and other factors all affect the treatment measures chosen for the patient. However, suitable comprehensive multidisciplinary treatment strategies for patients are required based on the overall consideration of various factors, to prevent the deterioration of the disease and to ensure that patients can maintain the long-term stability of the disease before they can undergo a liver transplantation, where possible.

In addition, in this study, the correlation between the patient's own factors and multidisciplinary treatment was studied. From the analysis of the occupational composition, the majority of patients in the MDT group were found to be white-collar workers, and the majority of patients in the traditional treatment group were farmers. The reason may be that the economic resources of the former are more stable, and this means they are more likely to have an economic guarantee for long-term treatment. From the etiological aspect, the majority of patients in the MDT group had hepatitis B-induced and C-induced cirrhosis. After antiviral treatment, most patients' conditions were controlled or even reversed, which led to an increase in their confidence, meaning they actively cooperated with the follow-up treatment and were more compliant. However, autoimmune, primary biliary cholangitis, cryptogenic, and alcoholic cirrhosis lack any effective etiological treatment, and are usually given symptomatic treatment. As patients lack belief in their treatment, the 5 -year survival rate and the compliance of the patients are lower than they are in the MDT group.

With gradually extend survival time in patients with cirrhosis, liver cirrhosis patients with variations death also produces change, the multidisciplinary treatment group the cause of death by cancer, infection, cirrhosis of liver disease cause of death rate increased, and the traditional treatment group cirrhosis of the cause of death is given priority to with hepatic failure, bleeding, infection, the dynamic changes of the need to pay attention to in clinical disease, timely intervention.

Twenty years ago, China lacked effective antiviral drugs. However, in the past two decades, with the continuous listing of new nucleoside drugs, the clinical application of entecavir, tenofovir ester, and other highly effective and highly resistant barrier drugs and direct-acting antivirals in patients with hepatitis C-induced cirrhosis has benefited more patients with hepatitis and cirrhosis, and the condition of approximately two-thirds of cirrhotic patients has been reversed. ${ }^{11-13}$ In recent years, the use of highly sensitive HBV DNA and HCV RNA detection technology has made it possible to screen patients with liver cirrhosis with low-level replication more accurately, and earlier and more accurately timed antiviral treatment has been the consequence. ${ }^{14,15}$ Clinicians are reminded that more sensitive nucleic acid detection is needed for patients with hepatitis B-induced cirrhosis, as this will be of great significance for decision-making and the implementation of key nodes such as initiating antiviral treatment, determining whether a complete response is achieved, and evaluating drug resistance. ${ }^{16-18}$ The Guidelines for Chronic Hepatitis B in China 2019 recommended antiviral therapy for decompensated hepatitis B-induced cirrhosis patients with positive or negative HBV DNA levels. This recommendation avoids the influence of different detection methods of HBV DNA nucleic acid on the treatment choice of clinicians.

Clinically speaking, it is necessary to follow the principle of the hierarchical and staged management of cirrhosis to facilitate dynamic follow-up management based on portal vein imaging, electronic gastroscopy, and laboratory tests. Increasing health education for patients and their family members, communicating with patients and their family members in a timely manner, and regularly reminding patients to attend follow-up examinations can improve the compliance of patients and solve relevant clinical problems to enable the early detection and treatment of cirrhosis.

\section{Conclusion}

An MDT to the treatment of cirrhosis can increase the survival rate of patients with cirrhosis and improve the quality of their lives. Improving patients' compliance and the early solving of related problems are essential for 
ensuring the improvement of the curative effect in patients with cirrhosis, and as they survive longer, their mortality spectrum changes.

\section{Ethics Approval and Consent to Participate}

This study was conducted in accordance with the declaration of Helsinki.This study was conducted with approval from the First People's Hospital of Lanzhou.A written informed consent was obtained from all participants.

\section{Funding}

Lanzhou Talent programs for Innovation and Entrepreneurship (2015-RC-73).

\section{Disclosure}

All authors have contributed significantly to the manuscript and declare that the work is original and has not been submitted or published elsewhere. None of the authors have any financial disclosure or conflicts of interest.

\section{References}

1. Ryder S. Guidelines for the diagnosis and treatment of hepatocellular carcinoma(HCC)in adults. Gut. 2003;52(Supple 3):iii1-iii8. doi:10.1136/gut.52.suppl_3.iii1

2. Sarin SK, Kumar M, Lau GK, et al.; Asian-Pacific Association for the Study of the Liver. Asian-Pacific clinical practice guidelines on the management of hepatitis B: a 2015 update. Hepatol Int. 2016;10:1-98.

3. de Franchis R. Expanding consensus in portal hypertension: report of the Baveno VI consensus workshop: stratifying risk and individualizing care for portal hypertension. $J$ Hepatol. 2015;63:743-752. doi:10.1016/j.jhep.2015.05.022

4. Tripathi D, Stanley AJ, Hayes PC, et al.; British Society of Gastroenterology. UK guidelines on the management of variceal haemorrhage in cirrhotic patients. Gut. 2015;64:1680-1704.

5. Garcia-Tsao G, Abraldes JG, Berzigotti A, Bosch J. Portal hypertensive bleeding in cirrhosis: risk stratification, diagnosis, and management: 2016 practice guidance by the American Association for the study of liver diseases. Hepatology. 2017;65(1):310-335. doi:10.1002/ hep.28906
6. Fagiuoli S, Bruno R, Debernardi Venon W; Italian Association for the Study of the Liver. Consensus conference on TIPS management: techniques, indications, contraindications. Dig Liver Dis. 2017;49 (2):121-137. doi:10.1016/j.dld.2016.10.011

7. Runyon BA; American Association for the Study of Liver Diseases. Introduction to the revised American Association for the Study of Liver Diseases Practice Guideline management of adult patients with ascites due to cirrhosis 2012. Hepatology. 2013;57(4):1651-1653. doi:10.1002/hep.26359

8. Miller CM, Quintini C, Dhawan A, et al. The international liver transplant society (ILTS) living donor liver transplant recipient guideline. Transplantation. 2017;101:938.

9. Caraceni P, Angeli P, Prati D; Italian Association for the Study of the Liver. AISF-SIMTI Position Paper: the appropriate use of albumin in patients with liver cirrhosis. Dig Liver Dis. 2016;48(1):4-15. doi:10.1016/j.dld.2015.11.008

10. Andriulli A, Tripodi A, Angeli P; Italian Association for the Study of the Liver. Hemostatic balance in patients with liver cirrhosis: report of a consensus conference. Dig Liver Dis. 2016;48(5):455-467. doi:10.1016/j.dld.2016.02.008

11. European Association for the Study of the Liver. EASL Clinical Practice Guidelines on nutrition in chronic liver disease. $J$ Hepatol. 2019;70:172-193.

12. Tripathi D, Stanley AJ, Hayes PC, et al.; Clinical Services and Standards Committee of the British Society of Gastroenterology. U. K.guidelines on the management of variceal haemorrhage in cirrhotic patients. Gut. 2015;64(11):1680-1704. doi:10.1136/gutjnl-2015309262

13. Terrault NA, Bzowej NH, Chang KM, et al. AALSD guidelines for treatment of chronic hepatitis B. Hepatology. 2015;63:261-283.

14. European Association for the Study of the Liver. EASL 2017 Clinical Practice Guidelines on the management of Hepatitis B virus infection. J Hepatol. 2017;67:370-398.

15. Terrault NA, Bzowej NH, Chang KM, Hwang JP, Jonas MM, Murad MH; American Association for the Study of Liver Diseases. AASLD guidelines for treatment of chronic hepatitis B. Hepatology. 2016;63(1):261-283. doi:10.1002/hep.28156

16. Sarin SK, Kumar M, Lau GK, et al. Asian-Pacific clinical practice guidelines on the management of hepatitis B: a 2015 update. Hepatol Int. 2016;10(1):1-98.

17. Terrault NA, Lok ASF, McMahon BJ, et al. Update on prevention, diagnosis, and treatment and of chronic hepatitis B: AASLD 2018 hepatitis B guidance. Hepatology. 2018;67(4):1560-1599.

18. European Association for the Study of the Liver. EASL Clinical Practice Guidelines for the management of patients with decompensated cirrhosis. J Hepatol. 2018;69:406-460.
Journal of Inflammation Research

\section{Publish your work in this journal}

The Journal of Inflammation Research is an international, peerreviewed open-access journal that welcomes laboratory and clinical findings on the molecular basis, cell biology and pharmacology of inflammation including original research, reviews, symposium reports, hypothesis formation and commentaries on: acute/chronic inflammation; mediators of inflammation; cellular processes; molecular mechanisms; pharmacology and novel anti-inflammatory drugs; clinical conditions involving inflammation. The manuscript management system is completely online and includes a very quick and fair peerreview system. Visit http://www.dovepress.com/testimonials.php to read real quotes from published authors. 